\title{
Chapitre IX \\ Des rôles multiples pour une femme régnante: différents genres pour la reine
}

L'Empire d'Autriche est une histoire de famille, et plus exactement d'une famille : celle des Habsbourg-Lorraine. Les seize enfants du couple impérial représentent une richesse et un atout indéniables, indispensables dans un contexte de crise dynastique $^{272}$. Aux côtés des couronnes, un discours de légitimation se met en place par l'intermédiaire des nombreux descendants de la nouvelle dynastie. Formant un type de portrait parmi d'autres mais non des moindres, les portraits de famille, avec Marie-Thérèse, François Étienne et leurs enfants, ainsi que les portraits de la souveraine avec son fils l'héritier Joseph enfant, sont pris en compte au cours du chapitre. Les couronnes des Habsbourg-Lorraine, incarnation des royaumes, forment un Empire, tenu et dirigé par une famille nombreuse, celle des HabsbourgLorraine.

Marie-Thérèse est l'élément d'une chaîne de transmission, tout en demeurant un élément fondateur de cette nouvelle dynastie des Habsbourg-Lorraine. Durant une période de crise dynastique, la légitimité de la souveraine doit être réaffirmée à l'aide du motif traditionnel de la mère et de l'enfant qui rappelle la Vierge Marie et l'enfant Jésus ${ }^{273}$. Cette figure renvoie aussi à la dimension sacramentelle de la dynastie et à ses ambitions impériales, ainsi qu'au premier devoir de toute monarchie héréditaire comme de toute épouse, celle d'instaurer et d'assurer une dynastie solide et prospère. Ce type de portrait offre de nouvelles perspectives et ressources en matière de représentation, et donc de pouvoir, à la Maison d'Autriche. En tant qu'épouse de l'empereur, Marie-Thérèse est impératrice. Bien plus, elle est la mère d'une nombreuse progéniture, parmi laquelle figurent de futurs empereurs.

La question des deux corps du roi est dépassée car Marie-Thérèse est un souverain fait femme, couronnée en tant que roi de Hongrie et de Bohême. Selon la

272 Braun, Eine Kaiserin und zwei Kaiser, p. 159. Bettina Braun explique bien dans son ouvrage que les seize enfants du couple étaient constamment présentés au public, en signe de « prospérité » de la famille impériale.

273 Sur les parallèles avec l'iconographie de la Vierge, voir Polleross, Das sakrale Identifikationsporträt, vol. 1, p. 150. Sur la piété mariale des Habsbourg et de Marie-Thérèse en particulier, voir Coreth, Pietas Austriaca, pp. 54-65 ; Ducreux, « Emperors, Kingdoms, Territories », pp. 284-285 ; Hawlik-van de Water, Der schöne Tod, pp. 83-89 ; Ingrao, Thomas, " Piety and Patronage », pp. 31-32 ; Karl, « Pietas Austriaca », p. 256 ; Linsboth, « Wünsche meine andacht zu verrichten », pp. 10-12, 16 ; Reb-Gombeaud, « Religion und Religiosität », pp. 25-26, p. 25 ; Vocelka, Heller, Lebenswelt, pp. 20-25.

2 Open Access. (c) 2022 Anne-Sophie Banakas, published by De Gruyter. (c) BY-NC-ND This work is licensed under the Creative Commons Attribution-NonCommercial-NoDerivatives 4.0 International License. https://doi.org/10.1515/9783110661804-013 
tradition monarchique, le roi est pourvu d'un corps immortel et politique, incarnation de l'idée d'État, et d'un corps périssable et naturel. Juridiquement, la souveraine est couronnée comme roi de Hongrie et de Bohême. Une ancienne tradition familiale rend d'ailleurs possible la désignation des femmes de la dynastie des Habsbourg à l'aide de titres masculins. Les archiduchesses d'Autriche, lorsqu'elles sont issues de la Maison d'Autriche, sont très souvent désignées comme archidux, Marie-Thérèse se nomme aussi archidux Austriae ${ }^{274}$. Elle est également couronnée comme roi de Hongrie en 1741, et de Bohême en 1743, et est ainsi parfois désignée sur les médailles comme Hungariae \& Bohemiae rex, mais on trouve aussi la désignation encore plus fréquente de regina, en particulier sur les monnaies ${ }^{275}$.

Malgré la Pragmatique Sanction, la position de Marie-Thérèse en tant que femme n'en est pas moins un obstacle, au début, en tout cas. Toutefois, au fil des années, cette position permet de tirer des types de portraits beaucoup plus marquants que si le souverain avait été un homme. Marie-Thérèse est à la fois roi couronné en tant que tel, tout en restant femme. La théorie des deux corps du roi, telle que l'a décrite Ernst Kantorowicz ${ }^{276}$, ouvre des pistes intéressantes mais ne peut en aucun cas être valable en toute situation. L'exposé de Kantorowicz qui s’applique principalement à l'époque médiévale n'offre qu'une structure d'explication imparfaite pour rendre compte de la complexité de la Monarchie composite autrichienne ${ }^{277}$.

Il est toutefois possible d'affirmer que le corps naturel de femme de la souveraine lui permet de magnifier son corps politique masculin. Marie-Thérèse utilise ainsi certains motifs typiquement féminins comme la maternité pour renforcer sa position en tant que souverain ${ }^{278}$.

274 Holzmair, « Maria Theresia », pp. 128-129.

275 Holzmair, « Maria Theresia », pp. 126-128.

276 Kantorowicz, The King's Two Bodies; voir note 19.

277 Voir les réflexions sur la relation de cette théorie au corps de la reine chez Edouard, Le corps d'une reine, p. 12 : « Le corps d'une reine, creuset à la fois d'une vie naturelle et d'une vie sacrée, offre une illustration parallèle de cette totalité de la 'vie nue' dans le politique. La reine est politique par la majesté sacralisatrice de son corps et par sa fonction biologique d'enfantement. [. . .] Ce corps de reine n'était pas celui du roi, le double corps juridique et physiquement mortel analysé par Ernst Kantorowicz, voire même le corps triple, selon Louis Marin, qui rajoutait aux deux précédents, le corps 'sacramentel' ou symbolique, c'est-à-dire celui de la représentation signifiante. » L'historienne se réfère à Marin, Le portrait du roi, pp. 14-19. Voir aussi sur la question de la maternité, les ouvrages de Badinter, Le pouvoir au féminin et Les conflits d'une mère.

278 Badinter, Le pouvoir au féminin, p. 330 ; Badinter parle même des trois corps de la reine. En plus du corps politique et naturel, Badinter évoque le « corps maternel qui perpétue la lignée » comme troisième corps. 
La question de la succession est un enjeu déterminant des héritages dynastiques et monarchiques au XVIII ${ }^{\mathrm{e}}$ siècle. Par la Pragmatique Sanction, Charles VI tente de faire respecter le principe de succession linéale cognatique. Malgré cela et jusqu'à sa mort survenue soudainement en 1740, Charles VI espère encore un héritier masculin. Même si Marie-Thérèse est en principe assurée de succéder à son père, la jeune archiduchesse n'est pas destinée au pouvoir. Cette succession en lignée féminine n'est pas la situation souhaitée ni recherchée mais seulement envisagée au cas où l'empereur n'aurait pas de fils qui pourrait hériter. Il s'agit de consolider le noyau familial étroit autour de Charles VI, composé principalement de son épouse Élisabeth Christine et de ses filles, Marie-Thérèse et Marie-Anne. En ce sens, la Pragmatique Sanction anticipe l'attention que Marie-Thérèse elle-même portera à sa propre famille, à son époux et à ses seize enfants.

Les symboles et insignes du pouvoir dont Marie-Thérèse hérite de sa famille servent d'instruments de légitimation. Cette légitimation a aussi lieu grâce à la nouvelle dynastie formée par sa nombreuse famille. La fertilité des HabsbourgLorraine est ainsi tout particulièrement mise en valeur par la représentation de l'héritier l'archiduc Joseph enfant à côté de Marie-Thérèse dans les années 1740, ainsi que dans les grands portraits de toute la famille impériale, réalisés dans les années 1750. Au cours de ce chapitre, nous continuons d'analyser les types d'images royales en nous demandant dans quelle mesure les enfants de la souveraine constituent un argument politique de première importance tout au long du règne. Comment la féminité de Marie-Thérèse, en soi un handicap ou du moins un désavantage, peut-elle dans ce cas aider la souveraine à s’imposer sur le plan politique en jouant sur le registre de la maternité ? Liée à la dignité impériale, la maternité incarne une forme de continuité.

En 1756 naît le dernier enfant de Marie-Thérèse, l'archiduc Maximilien, seizième enfant du couple impérial. Entre 1737 et 1756, presque chaque année voit la naissance d'un enfant. Comme les autres épouses de monarques avant elle, Marie-Thérèse accomplit sa fonction principale de femme et d'épouse : assurer une continuité dynastique tout en étant une épouse fidèle et féconde, garante de la descendance. Il importe donc à la souveraine de mettre en scène cette nouvelle dynastie, prospère et solide à la fois. Cette représentation familiale est commandée par Marie-Thérèse elle-même ainsi que par certaines grandes familles de la Monarchie comme par exemple les Schwarzenberg, les Pálffy, les Liechtenstein, qui installent ces portraits à côté de leurs propres portraits de famille.

Les différents types d'images, le genre du portrait de famille en particulier, établissent la légitimité et la continuité dynastique des Habsbourg-Lorraine, essentielle en ce milieu de XVIII ${ }^{\mathrm{e}}$ siècle après l'extinction des Habsbourg d'Espagne en 1701 et de la lignée masculine des Habsbourg d'Autriche en 1740. Le 
motif généalogique se retrouve de différentes manières, par exemple sous forme de galeries d'ancêtres, comme à l'abbaye de Melk qui commande en effet les portraits des souverains autrichiens allant des Babenberg jusqu'à Charles VI et Marie-Thérèse. Le principe dynastique et monarchique repose principalement sur la généalogie. ${ }^{279}$ Celle-ci englobe fondamentalement des hommes, Marie-Thérèse faisant figure d'exception dans cette longue généalogie masculine.

D’une manière générale, la question de la succession pose celle de la légitimité du pouvoir monarchique. La représentation de cette succession est un enjeu essentiel du règne de Marie-Thérèse. Après l'extinction de deux branches Habsbourg, en l'espace de quarante ans, il est plus que jamais nécessaire de représenter la force et la vitalité de la nouvelle dynastie des Habsbourg-Lorraine dont Marie-Thérèse et François Étienne sont les fondateurs, tout en poursuivant la tradition familiale des Habsbourg. Deux anciennes dynasties, les Habsbourg et les Lorraine, un temps menacées, se sont unies. Les anciens ducs de Lorraine ont en effet perdu leur duché au profit de Stanislas Leszczynski, beau-père du roi de France Louis XV.

En 1740, la continuité dynastique est fondamentalement ébranlée : guerre de Succession d'Autriche, remise en cause de la Pragmatique Sanction et soulèvements intérieurs lors de l'avènement de Marie-Thérèse. En raison de la précarité de la situation de la jeune souveraine, sous son règne, les représentations familiales des Habsbourg-Lorraine se multiplient pour légitimer la nouvelle dynastie des Habsbourg-Lorraine et pour réaffirmer le principe d'hérédité. C'est chose faite à la naissance de l'héritier Joseph en 1741 et lors de celles des autres fils du couple, comme les archiducs Charles et Léopold en 1745 et 1747. MarieThérèse apparaît alors comme « souverain » mais aussi comme la mère de l'héritier Joseph, que l'on destine très vite au titre de roi des Romains et à la fonction d'empereur du Saint Empire romain germanique.

Nous disposons de plusieurs portraits de Marie-Thérèse représentée avec son fils l'héritier Joseph enfant, de divers tableaux de toute la famille impériale au complet, sans compter les portraits de la souveraine et de son fils Joseph corégent après 1765 qui prennent la succession des portraits de Marie-Thérèse et de François Étienne. Ces portraits ne se limitent pas seulement au cercle viennois mais sont également exécutés sur initiative des élites elles-mêmes, comme l'atteste la commande de l'abbaye bénédictine de Seitenstetten en Basse-Autriche.

Le principe d'hérédité est étendu à toute la famille de Marie-Thérèse, à la familia augusta, aux enfants, garçons comme filles, qui participent à la politique dynastique de Marie-Thérèse par leurs mariages. La fertilité de la nouvelle

279 Barta, Familienporträts, pp. 52-61. 
dynastie est mise en scène et présentée en exemple, rapprochée de l'idéal de la famille aristocratique, mais aussi, surtout vers la fin du règne, de la famille bourgeoise. Le couple impérial et ses enfants forment et offrent l'image d'une famille unie. Des écrits de l'époque soulignent la fertilité de la nouvelle dynastie. Ainsi le poème honorifique de Franz Christoph von Scheyb, la Theresiade, cité plus haut au sujet du couronnement hongrois, est dédié à Marie-Thérèse à l'occasion des festivités liées à la naissance de son second fils, Charles Joseph (1745-1761). Cette seconde naissance masculine témoigne de la consolidation de la lignée des Habsbourg-Lorraine ${ }^{280}$.

Par la représentation de son nombre et de son unité, cette dynastie en crise montre ainsi son droit à durer en arborant la première des richesses et des garanties de toute famille, une nombreuse descendance, particulièrement indispensable dans le cadre d'un discours dynastique. Aux côtés des portraits, beaucoup de gravures mettent en scène un arbre généalogique de la famille de la souveraine centré sur descendance ${ }^{281}$. Ces arbres généalogiques rappellent à leur manière les pièces familiales dédiées aux Habsbourg-Lorraine, aux enfants et aux petits-enfants du couple impérial. La famille de Marie-Thérèse fonde une nouvelle lignée ${ }^{282}$.

Dans les nouveaux espaces consacrés à la représentation impériale et à la célébration dynastique dans les palais impériaux rénovés des années 1760, les membres de la famille des Habsbourg-Lorraine ont leurs portraits. Les portraits de chaque membre sont exposés dans des salles comme dans celle des Géants de la Hofburg d'Innsbruck. Là, Marie-Thérèse est peinte en Grand Maître de l'Ordre de Saint-Étienne. Au château de Prague, elle est représentée en habit de couronnement de roi de Bohême, tandis qu'au château de Buda, la souveraine apparaît en roi de Hongrie. Marie-Thérèse n'est pas seule à la tête de ses pays, elle est accompagnée de sa famille, de son époux et de leurs seize enfants. Dans de tels espaces, la souveraine est entourée de François Étienne et de Joseph, l'empereur et le futur roi des Romains.

Jusqu'au XVII ${ }^{\mathrm{e}}$ siècle, les ouvrages et arbres généalogiques commandés par la cour, ou destinés à son usage, attribuaient volontiers des origines lointaines et prestigieuses aux Habsbourg, remontant très souvent aux Troyens ou à Charlemagne $^{283}$. Au temps de Marie-Thérèse, de telles affirmations sont beaucoup moins courantes. L'érudition historique et les besoins de légitimation ne sont

280 Übelleitner, Die Tugenden Maria Theresias, p. 40; Scheyb, Theresiade.

281 Barta, Familienporträts, pp. 56-57.

282 Barta, Familienporträts, p. 44.

283 Lhotsky, "Apis Colonna »; voir aussi Brockmann, " Das Bild des Hauses Habsburg », pp. 30-37; Coreth, Österreichische Geschichtschreibung, pp. 30-42; Kovács, « Die Apotheose des Hau- 
plus les mêmes, sans cesser d'exister pour autant. On insiste désormais plutôt sur l'origine commune entre les Habsbourg et les Lorraine, les deux maisons ayant l'Alsace comme berceau familial. Les liens de parenté entre la Maison de Lorraine et celle d'Autriche sont ainsi régulièrement rappelés, en particulier lorsqu'il s'agit d'élire et de faire couronner François Étienne comme empereur. Le plus remarquable des ouvrages de ce genre est constitué par les quatre volumes des Monumenta Augustae Domus Austriacae du bénédictin Marquard Herrgott, réalisés entre 1750 et $1772^{284}$.

Les portraits participent également à ce projet généalogique, en particulier lorsqu'ils mettent en scène les divers membres de la famille des HabsbourgLorraine, notamment à travers la figure de la mère. Les portraits de MarieThérèse évoquent aussi cette figure maternelle à la tête de la maison dynastique des Habsbourg-Lorraine; mère d'une nombreuse descendance, mère de ses peuples. Dans la société traditionnelle ordonnée de manière patriarcale, societas civilis, la maison est l'unité structurelle la plus importante. La maison et les territoires sous souveraineté de la dynastie sont alors unis par la personne du souverain, le père, à qui est attribué un rôle à la fois protecteur, paternel et nourricier. En tête de sa dynastie, le souverain est également le père de ses peuples $^{285}$. En s'appuyant sur ce complexe de métaphores, mais aussi en l'adaptant à son cas particulier, Marie-Thérèse est à la fois la mère et le père de ses peuples, ou une mère appelée à jouer un rôle protecteur de père ${ }^{286}$.

$\mathrm{Au}$ cours des dernières décennies, les études d'Ilsebill Barta ${ }^{287}$ ou encore de l'Américain Michael Yonan ${ }^{288}$ ont abordé la question de la représentation de Marie-

ses Österreich », p. 59; Seitschek, « Adel und Genealogie », pp. 81-87; Winkelbauer, Ständefreiheit und Fürstenmacht, vol. 1, pp. 253-257.

284 Herrgott, Monumenta Augustae Domus Austriacae. Sur l'œuvre et son auteur, voir Benz, Zwischen Tradition und Kritik, pp. 447-454; Ortner, Marquard Herrgott, pp. 64-69; Seitschek, "Adel und Genealogie », p. 84.

285 Boldt et al., "Monarchie », pp. 175-176. On y cite entre autres Conring, « Dissertatio de ortu et mutationibus regnorum », p. 1068 : « regnum scilicet proprie dictum, quando unus omnia in sua manu, et subditos tenet imperio, non herili, sed civili ad subditorum potius, quam suam utilitatem, ut bonus Paterfamilias praeest suae domui ».

286 Sur l'aspect souverain et maternel à la fois de Marie-Thérèse, voir notamment l'ouvrage cité de Regina Schulte, Der Körper der Königin, pp.18-20. Regina Schulte souligne que MarieThérèse joue en permanence sur « la transgression entre son corps politique et son corps naturel », en particulier lors du couronnement hongrois. Regina Schulte insiste sur le fait que la souveraine sait parfaitement mettre en valeur et utiliser, d'un point de vue politique, son corps maternel, voir p. 20.

287 Barta, Familienporträts.

288 Yonan, Empress Maria Theresa; Yonan, «Conceptualizing the Kaiserinwitwe »; Yonan, « Modesty and Monarchy ». 
Thérèse. Dans ces ouvrages, les notions de maternité, de fertilité et de famille sont mises en valeur, tout particulièrement par Barta. Nous ne tentons pas de refaire le travail déjà accompli par les auteurs qui se sont attachés à décrire de manière précise les objets et les symboles représentés dans les portraits de famille de Marie-Thérèse. Mentionnons rapidement certains symboles de fertilité et d'abondance présents comme par exemple le palmier ou le vase dans des tableaux de famille de Martin van Meytens ${ }^{289}$.

Première à s'être consacrée au portrait de Marie-Thérèse, Ilsebill Barta s'est principalement attachée à l'étude du portrait familial chez les Habsbourg, tout particulièrement pour le règne de Marie-Thérèse. Il existe une tradition ancienne du portrait familial chez les Habsbourg, si l'on pense par exemple au portrait de Maximilien $\mathrm{I}^{\mathrm{er}}$ avec sa famille, réalisé vers 1515-1516 par Bernhard Strigel $^{290}$ ou à celui, non moins célèbre, de l'empereur Maximilien II avec sa femme et ses enfants par Arcimboldo remontant aux environs de $1567^{291}$. Barta montre l'évolution du genre au cours du siècle des Lumières. Le portrait de famille prend de plus en plus comme modèle la famille des Habsbourg-Lorraine, et se rapproche progressivement du modèle bourgeois de la famille formée par les parents et les enfants. Quant à Werner Telesko, il évoque la représentation familiale de Marie-Thérèse comme un argument dynastique ${ }^{292}$.

Le concept généalogique et politique de la Domus Austria est soumis à un profond changement au profit d'une nouvelle mise en valeur de la nombreuse et brillante famille fondée par Marie-Thérèse et François Étienne ${ }^{293}$. L'image de la famille impériale est alors principalement focalisée autour du couple impérial et de ses enfants ${ }^{294}$. Soulignons que les portraits à huile, officiels, de la famille impériale sont plus rares que les gravures et les médaillons ${ }^{295}$. Dans notre corpus de tableaux, nous n'avons en effet que quelques portraits de la famille complète, c'est-à-dire le couple impérial et leurs enfants, exécutés pour la plupart par Martin van Meytens et son atelier (P 140, P 141, P 142, P 143, P 144, P 145, P 146, P 216).

289 Barta, Familienporträts, pp. 62-69, 73-83, 91-97.

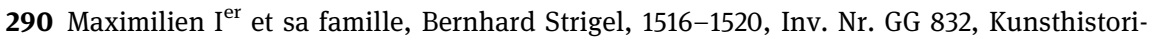
sches Museum Vienne. Voir note 38, introduction générale.

291 Giuseppe Arcimboldo, portrait de famille de Maximilien II, 1567, Kunsthistorisches Museum, Vienne. Götz, Die Künstler der Kaiser: Von Dürer bis Tizian, p. 16.

292 Telesko, Maria Theresia, pp. 48-49.

293 Telesko, Maria Theresia, p. 48, évoque le terme de Kernfamilie.

294 Barta, Familienporträts, pp. 39-51; Telesko, Maria Theresia, p. 48.

295 Les supports de communication en gravures et en médaillons se multiplient et permettent de répandre plus largement l'image de la fertilité et de la force numérique de la nouvelle dynastie des Habsbourg-Lorraine ; voir Stollberg-Rilinger, Maria Theresia, p. 464. 
Mère, Marie-Thérèse n'en demeure pas moins souverain. Sa représentation propose aussi différents rôles et une image équestre. La féminité n'est pas seulement liée à la maternité mais aussi à la diversité des représentations du corps de Marie-Thérèse.

\section{L'existence d'un héritier : un argument politique crucial}

« Bella gerant alii, tu felix Austria nube.

Nam quae Mars aliis, dat tibi diva Venus ${ }^{296}$

Traditionnellement, selon le dicton, les Habsbourg prisent davantage les unions maritales que les guerres pour s'agrandir et étendre leurs royaumes -ou du moins aiment-ils à se présenter de la sorte. Les unions sont ainsi représentées dans une image où les enfants, en particulier les héritiers, sont mis en avant. L'image familiale Habsbourg-Lorraine apparaît comme une image de propagande relayée par les écrits ${ }^{297}$.

Un mythe s'est développé dans la postérité autour de la figure de MarieThérèse qui diffuse l'image d'une souveraine, mère de ses peuples ${ }^{298}$. Un des premiers témoignages de cette transfiguration de Marie-Thérèse dans sa fonction de mère est rapporté par une apologie écrite par Joseph de Sonnenfels le 13 mai 1762 à l'occasion du quarante-cinquième anniversaire de la souveraine. Sonnenfels y évoque Marie-Thérèse comme « la mère de ses peuples ${ }^{299}$. Disposant d'une fonction pédagogique, la souveraine fait alors partie intégrante d'un programme d'éducation. Sonnenfels esquisse l'image d'une femme engagée qui s'efforce de mettre en place une certaine forme d'absolutisme éclairé ${ }^{300}$. Il utilise le motif de la maternité dans le sens de modèle et d'exemple.

296 La traduction allemande de cette citation latine est la suivante : « Kriege führen mögen andere, du glückliches Österreich heirate, Denn was Mars (den) anderen (verschafft), gibt dir die göttliche Venus. Laisse les autres faire la guerre, toi, heureuse Autriche, marie-toi [. . .]»; Voir Klecker, «Bella gerant alii », p. 33.

297 Sur la question des femmes Habsbourg et de leur mariage, voir aussi Patrouch, " Bella gerant alii », p. 25. Les filles Habsbourg constituent au cours de l'époque moderne un élément particulièrement important pour l'établissement et la consolidation de leur propre dynastie. Épouses et mères, ces femmes Habsbourg n'en continuent pas moins, même mariées, de soutenir la politique de leur dynastie d'origine.

298 Telesko, Maria Theresia, p. 114.

299 Vocelka, Glanz und Untergang, p. 30.

300 Telesko, Maria Theresia, pp. 114-115: « Die Hausmutter gelobet, das Beispiel der Fürstin, als eine zärtliche und getreue Gattin, als eine sorgende Mutter, als eine liebreiche Frau nachzuahmen » 
Des récits plus ou moins légendaires mettent également l'accent sur la dimension maternelle de Marie-Thérèse. Le plus répandu est sans doute celui du Vitam et Sanguinem pro rege nostro Maria Theresia, à Presbourg en 1741, où Marie-Thérèse arrive éplorée devant la diète hongroise avec l'archiduc Joseph enfant dans ses bras et supplie les magnats de venir la secourir. Émus à la vue de cette image, les preux Hongrois lèvent d'un seul chef leur sabre pour jurer aide et fidélité à Marie-Thérèse, se déclarant prêts à donner leur vie et leur sang pour leur « roi ». Si cette scène est fictive quant à la présence de l'enfant selon Barbara Stollberg-Rilinger, ce serait Voltaire qui aurait inventé ce détail elle devient très populaire parmi les contemporains ${ }^{301}$. De telles anecdotes renforcent l'idée que l'image de Marie-Thérèse avec son fils devient un élément artistique, correspondant à une notion clé de référence et même de propagande, soulignant la continuité et l'avenir dynastique qu'il s'agit d'assurer.

En 1742, une gravure sous forme de tract, mise en circulation en Hollande, décrit, commente et représente une scène qui se serait déroulée en Autriche alors que les troupes de l'électeur de Bavière occupaient toute la HauteAutriche et que la situation semblait désespérée. La souveraine aurait alors écrit une lettre à son maréchal de camp, le comte Ludwig Andreas Khevenhüller, qui commandait l'armée chargée de la défense du pays. Le futur empereur François $\mathrm{I}^{\mathrm{er}}$ aurait personnellement remis la missive. À la lettre s'ajoute un portrait de Marie-Thérèse et de son fils, auquel la souveraine fait référence en écrivant ces mots : « Tu as sous les yeux une jeune reine abandonnée de tous. Que penses-tu qu'il adviendra de son destin et de celui de son enfant ? Regarde ta princesse dans les yeux $»^{302}$.

L'image montre la réaction du maréchal qui rassemble ses soldats devant la tente, la lettre dans la main droite, les portraits dans la main gauche, et présente alors l'image royale à tous les hommes qui sortent précipitamment des tentes pour voir leur souveraine. Le texte inscrit sur le tract rapporte la manière dont les soldats brandissent leurs sabres, et renouvellent leur serment de fidélité. Cette scène, en partie légendaire, constitue toutefois un témoignage supplémentaire de l'importance accordée au portrait de la souveraine et de son héritier, autant de motifs maternels répandus et largement diffusés.

cité par Telesko, Maria Theresia, p.114, d'après Mauser, « Maria Theresia. Mütterlichkeit: Mythos und politisches Mandat », pp. 77-97, p. 86.

301 Stollberg-Rilinger, Maria Theresia, pp. 90-92; voir aussi Fazekas, Ujváry, " Katalog », p. 151.

302 Warnke, « Politische Ikonographie », pp. 25-26; voir aussi Barta, Familienporträts, pp. 72-73; Schmitt-Vorster, Joseph II, pp. 8-9; voir aussi note 1, introduction générale. 
Si ces scènes n'entrent pas dans les portraits officiels, les années 1740 marquées par la guerre de Succession d'Autriche sont bien l'époque des tableaux montrant Marie-Thérèse avec l'archiduc Joseph enfant, une première phase pour ainsi dire de la représentation familiale. Ces tableaux de Marie-Thérèse avec Joseph enfant se trouvent particulièrement en Bohême.

Ce n'est pas une coïncidence si trois portraits avec l'archiduc Joseph enfant se trouvent à Prague, deux au Musée de la ville (P 78, Figure 31, P 79) et un autre à l'abbaye de Břevnov $(\mathrm{P} 77)^{303}$. Comme nous l'avons vu lorsqu'il s'agissait de la représentation de la couronne de Bohême, ces portraits exécutés entre 1743 et 1745 interviennent à un moment et à un endroit particulièrement crucial pour témoigner de la force de la nouvelle dynastie. À l'époque des couronnements de Marie-Thérèse comme roi de Bohême et de son époux comme empereur, on indique la perspective pour l'avenir en soulignant que la dynastie des Habsbourg-Lorraine dispose désormais d'une descendance masculine avec l'archiduc Joseph, représenté comme futur roi des Romains.

La représentation avec l'héritier Joseph enfant renoue avec un motif pictural traditionnel des souveraines, en particulier des souveraines Habsbourg, mais aussi de toutes les reines et plus encore de toutes les mères. Il a été montré que la maternité est un état politique dans la France ${ }^{304}$ et dans l'Europe de l'époque moderne, liée à la stabilité et à la prospérité de l'État mais aussi à la prospérité des dynasties pour lesquelles les femmes, et notamment les mères, sont amenées à jouer un rôle considérable $\mathrm{e}^{305}$. Une dynastie et une Maison prospères dépendent donc étroitement de la mère.

$\mathrm{Au}$ début du règne, vers 1743 , les portraits de Marie-Thérèse accompagnée de l'archiduc Joseph enfant sont particulièrement, mais pas uniquement, destinés à un public issu de Bohême, façon peut-être de rassurer les élites de ce royaume concernant la solidité de la nouvelle dynastie durant une période particulièrement critique. Comme nous l'avons déjà évoqué, la couronne impériale est également souvent peinte dans de tels portraits, manière de souligner que la nouvelle famille des Habsbourg-Lorraine reste attachée à la dignité impériale. Le grand portrait de Marie-Thérèse et de l'archiduc Joseph enfant, réalisé

303 Menzel, «Ein Blick in die barocke Welt », p. 105; Vilímková, Preiss, Ve znamení břevna a růzí, pp. 260, 307.

304 Germann, Picturing Marie Leszczinska, p. 3 et pp. 99-128.

305 Cruz, «Introduction », p. 12: « [. . . ] there can be no doubt that fecundity played a vital role in Habsburg women's lives, as it meant the dynasty's very survival. [. . . ] contemporary culture valued royal women first and foremost for their childbearing capability ». Sur la maternité et la fécondité chez les femmes Habsbourg, voir par exemple Cruz de Carlos Verona, « Giving Birth at the Habsburg Court »; Mitchell, « Habsburg Motherhood ». 
vers 1743-1745, est conservé au Musée de la ville de Prague (P 78, Figure 31). Le peintre qui réalise ce portrait s'est probablement inspiré du portrait d'Auerbach conservé à l'abbaye de Břevnov (P 77).

Après avoir représenté Marie-Thérèse dans le portrait réalisé par Andreas Möller (P 1, Figure 1$)^{306}$, en jeune femme gracieuse et potentiellement mère, à l'aide de symboles de fertilité comme les fleurs, les portraits la montrent de plus en plus aux côtés de ses enfants et en premier lieu de son fils aîné. Le médaillon de l'archiduc Joseph qui se trouve au-dessus du portrait de Marie-Thérèse commandé par l'hôtel de ville de Vienne en 1744 l'atteste (P 12) ${ }^{307}$. Ce tableau dispose de trois niveaux de représentation : en haut est représentée la couronne impériale familiale, suspendue au-dessus d'un médaillon de l'archiduc Joseph enfant, le tout surplombant la représentation en pied de Marie-Thérèse ${ }^{308}$. Ces trois niveaux forment une unité verticale et donnent l'impression de linéarité dynastique et monarchique entre Joseph, Marie-Thérèse et la dignité impériale. L'avenir de la dynastie et de l'Empire est désormais entre les mains d'un descendant de la dynastie des Habsbourg-Lorraine.

Dans le portrait de Seitenstetten en Autriche (P 80, Figure 33) ${ }^{309}$, le jeune archiduc Joseph est représenté à l'âge de trois ou quatre ans. Il est vêtu d'un costume hongrois et porte un pendentif de l'Ordre de la Toison d'Or, il pose sa main sur l'orbe, l'autre dans celle de sa mère. Notons que la couronne impériale de Nuremberg est représentée non loin de Joseph ${ }^{310}$.

Les images de la femme et de la mère sont mises en scène afin de défendre un certain nombre de messages politiques et dynastiques. Dans le cas de MarieThérèse, il s'agit de souligner la continuité dynastique entre les Habsbourg et les Habsbourg-Lorraine, en particulier dans les années 1740, par l'intermédiaire de la maternité. L'association de Marie-Thérèse, de l'archiduc Joseph enfant et de la couronne impériale familiale renforce cette impression de continuité impériale familiale liée à la Maison d'Autriche.

306 Ce portrait est déjà discuté dans le chapitre VIII.

307 Voir note 375, chapitre V.

308 Yonan, Empress Maria Theresa, pp. 26-28.

309 Merci au Père Martin Mayrhofer pour son aide concernant le portrait de Seitenstetten.

310 Voir note 227, chapitre III. 


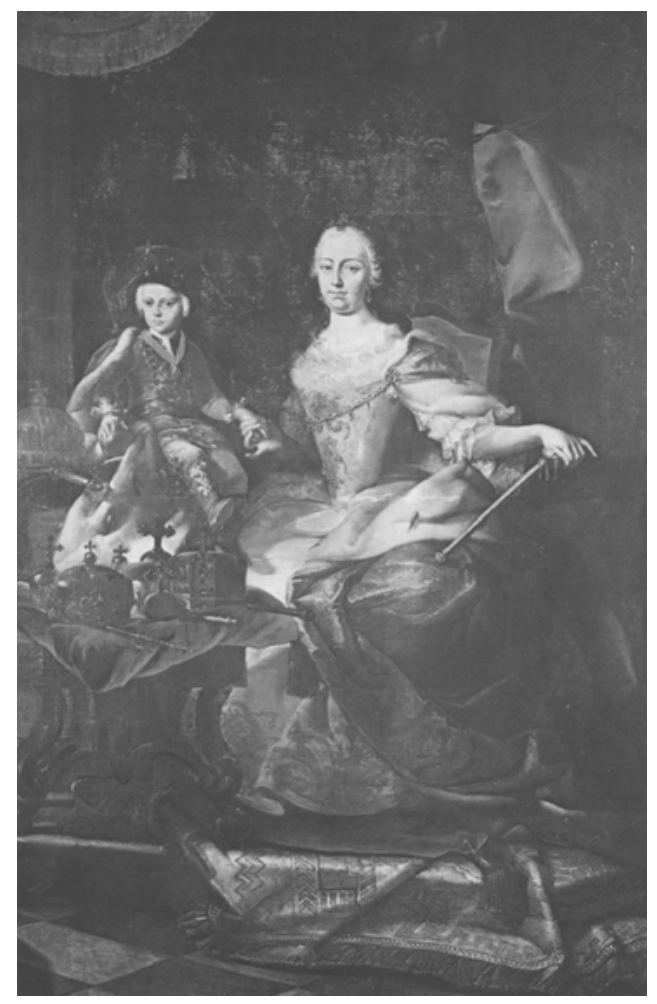

Figure 33: Martin Johann Schmidt, “Martin Joh. Schmidt fec. 1745”, Marie-Thérèse et l'archiduc Joseph enfant, année 1745, $239 \times 157 \mathrm{~cm}$, abbaye de Seitenstetten, Autriche. Le tableau se trouve dans la première pièce de l'abbaye d'été du couvent.

\section{Les années 1750 ou la décennie des grands portraits de famille}

Par la suite, les portraits de famille réalisés par le peintre officiel Martin van Meytens dans les années 1750 sont tout particulièrement peints à la demande de Marie-Thérèse et mettent l'accent sur l'ensemble et l'unité des HabsbourgLorraine. La composition familiale officielle la plus connue est celle avec la vue sur le parc du château de Schönbrunn où les deux parents, Marie-Thérèse et François Étienne, se répartissent de part et d'autre de la surface ${ }^{311}$. Comme

311 Depuis le XVI ${ }^{\mathrm{e}}$ siècle, il existe deux types de composition de la famille réunie, un premier type qui privilégie les parents au centre du tableau et l'autre où les parents sont représentés 
pour les autres types de portraits, des copies sous forme de peintures et de gravures de cette version originale sont ensuite envoyées dans de nombreuses cours européennes ${ }^{312}$.

Dans les factures de la chambre des comptes de la cour ou dans les dossiers de l'Académie viennoise, en particulier dans les écrits laissés par le chancelier d'État Kaunitz et adressés à la souveraine dans les années $1770^{313}$, une augmentation assez spectaculaire des portraits de famille est enregistrée entre les années 1750 jusque dans les années 1770. Des esquisses des grands portraits de familles sont alors produites en de multiples exemplaires par des dizaines de peintres, à en croire du moins ce qu'en rapporte Kaunitz.

Arguments politiques de choix, les portraits de famille mettent en valeur la principale richesse de Marie-Thérèse. Les tableaux de Meytens positionnent les fils du côté de Marie-Thérèse et les filles du côté de François Étienne. MarieThérèse reste le centre du pouvoir vers lequel François Étienne dirige sa main gauche. Les archiducs Joseph et Charles désignent également la personne de Marie-Thérèse. Cette dernière accepte de son côté leur geste et le redirige vers elle $^{314}$. Dans ce type de portrait, le rôle dominant de Marie-Thérèse est rendu visible par la composition, ainsi que les titres des deux époux par la présence des couronnes. En même temps, il apparaît clairement que Marie-Thérèse a accompli la fonction essentielle d'une femme, qui est plus d'une souveraine, engendrer une nombreuse descendance d'héritiers mâles ainsi que de nombreuses filles, potentielles épouses de princes européens. La représentation de la famille de Marie-Thérèse devient une valeur de référence.

Cette représentation, particulièrement réussie, devient le portrait officiel de la famille impériale dans les années 1750 tandis que la situation politique, diplomatique et économique se stabilise. Martin van Meytens et son atelier sont donc amenés à reproduire ce motif au gré des nombreuses et régulières naissances impériales. Chaque fois, les nouveaux enfants sont rajoutés au centre de la composition sans modifier fondamentalement l'agencement général des mo-

de chaque côté. La mise en scène décentralisée remonte plutôt au cercle culturel du nord tandis que la composition centralisée aurait une origine italienne ; voir Barta, Familienporträts, p. 97 ; concernant les portraits de famille, voir aussi Roeck, Das historische Auge, p. 181.

312 Barta, Familienporträts, p. 96.

313 Voir partie I, chapitre I. AVA Unterricht Akademien StHK Teil 1 A 757 Wien, Akademie der bildenden Künste, fo. 21, à Vienne 17 mars 1775; lettre de Kaunitz à S. M. l'Impératrice Reine. « Madame ! J'ai l'honneur d'envoyer à Votre Majesté des Esquisses pour les grands portraits de famille qu'elle m’a commandés. Ils sont faits par 10 personnes différentes [. . .] ».

314 Barta, Familienporträts, p. 92; voir aussi Banakas, " Die zwei Körper der Herrscherin », pp. 78-79, 87; Polleross, « Das frühneuzeitliche Bildnis als Quelle », p. 1017; Hertel, Banakas, « Die Gestik Maria Theresias », p. 210. 
dèles ni le décor en arrière-plan ${ }^{315}$. Durant ces années clés en matière de reconquête du pouvoir sur la scène internationale, au moins quatre représentations de la famille impériale au complet par Meytens mettent en scène la famille des Habsbourg-Lorraine $\mathrm{e}^{316}$. La première, exposée originellement à la Hofburg d'Innsbruck, date de 1751 ou 1752 ; le couple impérial est peint avec neuf de ses enfants $(\mathrm{P} 140)^{317}$. La seconde, exposée au château de Schönbrunn, remonte à 1754 et montre onze enfants (P 141). En 1755, la naissance de Marie-Antoinette entraîne une nouvelle version du portrait de famille avec douze enfants (P 145). Ce portrait est aujourd'hui au château de Versailles ${ }^{318}$. En 1756, un dernier tableau est réalisé. Ce portrait, conservé au palais Pitti à Florence (P 216) ${ }^{319}$, est peint après la naissance de l'archiduc Maximilien, dernier et seizième enfant du couple impérial. Il apparaît à son tour dans le berceau. Les grands portraits de famille utilisent une image de Marie-Thérèse que l'on retrouve dans beaucoup de portraits individuels de la souveraine, notamment dans les années 1750. L'esquisse d'une tête ou d'un buste original est alors copiée avec différentes poses, différents habits et accessoires pour les divers portraits impériaux selon les besoins et les volontés des commanditaires.

Dans ces grands portraits de famille, la représentation des enfants, entre l'empereur du Saint Empire et Marie-Thérèse en sa fonction de roi de Hongrie et de Bohême, témoigne de l'héritage familial. Ces portraits correspondent au type traditionnel des portraits de familles. Ils s'inscrivent dans la tradition des tableaux dynastiques, notamment ceux d'Arcimboldo avec sa représentation de l'empereur Maximilien II et des siens ${ }^{320}$.

Il arrive ainsi que des peintures plus intimes et familiales respectent un peu moins l'ordre d'organisation et de répartition des enfants. Dans les châteaux de la noblesse, par exemple, comme au château de Konopiště en actuelle République tchèque $(\mathrm{P} 144)^{321}$, on retrouve exactement ce type de représentation, avec

315 Salmon, « Martin van Meytens », p. 68.

316 Lisholm, Martin van Meytens, pp. 66, 104, 130-131; Salmon, " Martin van Meytens »; Schütz, « Martin van Meytens », p. 184.

317 Banakas, «Gruppenbildnis ».

318 Salmon, « Martin van Meytens », p. 68.

319 Barta, Familienporträts, p. 94; Lisholm, Martin van Meytens, p. 104. Cette composition sera reprise encore jusqu'à la mort de François Étienne ; voir Schütz, « Martin van Meytens ».

320 Vienne, Kunsthistorisches Museum, Gemäldegalerie, Inv. Nr. 3448. Voir Kuster, « Maria Theresia und Franz I. Stephan mit elf Kindern », p. 232.

321 Ce portrait est une représentation traditionnelle de la famille impériale au côté des couronnes, les enfants sont répartis selon le schéma classique, les filles auprès du père et l'héritier Joseph est à côté de la souveraine, le chef d'État par qui l'hérédité politique se transmet, toutefois l'archiduc Léopold est quand même peint à côté de l'empereur. La famille de l'archiduc François 
toutefois quelques nuances qui diffèrent en ce qui concerne la mise en scène. Dans ces répliques des grandes œuvres de Meytens, attribuées en bien des cas au cercle de Meytens, la famille impériale apparaît de manière un peu plus détendue, les couronnes sont parfois absentes, ou reléguées en arrière-plan. Dans le portrait de Konopiště, l'archiduc héritier Joseph est de tout de même représenté près de Marie-Thérèse. Un portrait très similaire à celui de Konopiště se trouve dans les collections de Basse-Autriche ${ }^{322}$.

À côté de ce type d'image familiale assez majestueuse, il en existe d'autres qui suivent des modèles différents. C'est le cas d'un portrait, probablement issu de l'atelier de Meytens et réalisé vers 1758, aujourd'hui au château de Gödöllő en Hongrie (P 146). Dans cette huile sur toile, de dimensions relativement petites, les parents sont au centre, côte à côte sur un canapé, tandis que les enfants sont répartis dans toute la pièce sans ordre véritable, en train de jouer de la musique sur divers instruments. La composition avec les parents assis au centre s'inspire d'un schéma traditionnel de représentation familiale habituelle à la cour du XVIII ${ }^{\mathrm{e}}$ siècle $^{323}$.

La structure du portrait avec la répartition du couple, l'époux à gauche, l'épouse à droite, les enfants au centre ou répartis des deux côtés du couple, et à leurs pieds un chien, symbole de fidélité conjugale ${ }^{324}$, est la même que celle du portrait de la famille impériale par Meytens ${ }^{325}$.

L'ambiance intime de ce tableau, cependant, participe au genre bourgeois qui apparaît alors à la cour, très prisé par la haute aristocratie. Le sens d'une telle image consiste à mêler l'importance de la lignée dynastique avec l'inclination bourgeoise pour le portrait de famille, plus intimiste, simple et dépouillé ${ }^{326}$. Les évènements quotidiens ainsi que les situations intimes bourgeoises deviennent des thèmes de représentation.

Ferdinand vivait très souvent dans le château de Konopiště en Bohême avant la première guerre mondiale. Cette demeure appartenait à la famille Chotek. L'épouse de François Ferdinand n'était autre que Sophie Chotek, descendante des conseillers de Marie-Thérèse. Ce portrait de la famille impériale pourrait donc provenir de la famille Chotek. Il pourrait aussi avoir été facilement amené à Konopiště par François Ferdinand, en provenance des collections impériales.

322 Lechner, «Franz I. Stephan, Maria Theresia und ihre Kinder ».

323 Barta, Familienporträts, p. 96.

324 Concernant le chien comme symbole de fidélité conjugale, voir Barta, Familienporträts, p. 92.

325 Le portrait familial du duc Léopold $\mathrm{I}^{\mathrm{er}}$ de Lorraine, le père de François Étienne, est souvent mentionné comme modèle. Il s'agit d'un tableau réalisé par Jean Renauld vers 1720, mesurant 55,5 x 71,5 cm, conservé au Kunsthistorisches Museum de Vienne. Voir notamment Barta, Familienporträts, pp. 95-96.

326 Buzási, Régi Magyar Arcképek, Alte ungarische Bildnisse, pp. 61-62. Portrait 24. 
Manifestation politique en vue de légitimer une nouvelle dynastie, le genre du portrait de famille reflète aussi le goût de l'époque pour ce type de portrait. La famille n'est plus seulement comprise au sein d'une grande lignée d'ancêtres, elle est principalement centrée sur le noyau familial, et composée des parents et de leurs enfants ${ }^{327}$.

\section{Le cas particulier de la représentation de la souveraine et de ses fils après la mort de François Étienne en 1765}

En Moravie, on attribue au peintre Franz Anton Palko, un portrait de famille de très grandes dimensions représentant vers 1766-1768 Marie-Thérèse et trois de ses fils, Léopold, Ferdinand et Maximilien (P 143, Figure 34) ${ }^{328}$. Après la mort de François Étienne en 1765, ses fils assurent et se partagent ses fonctions. Léopold et Ferdinand sont grand-duc de Toscane pour l'un et gouverneur général de la Lombardie pour l'autre, et Léopold devient empereur après la mort de son frère Joseph. Quant au dernier fils et enfant de la famille impériale, l'archiduc Maximilien, il sera nommé archevêque électeur de Cologne ${ }^{329}$.

Les personnages sont peints de manière grandiose et impressionnante, quelque peu surélevés sur un piédestal, entourés par un précepteur vêtu de noir et par des conseillers. Le buste de François Étienne est installé en arrière-plan, signe que le portrait a certainement été réalisé après la mort de ce dernier en 1765. Une couronne est portée au-dessus de la tête de Léopold, le désignant comme possible successeur de son père en Toscane. En arrière, les différentes couronnes sont représentées, la couronne impériale ainsi que celles de Hongrie et de Bohême. Dans le même château de Valtice est exposé un autre tableau, apparemment un pendant au premier, représentant Joseph II et sa femme, Marie-Josèphe de Bavière ${ }^{330}$. Cette paire de tableaux apparaît donc comme une version élargie de la combinaison habituelle après la mort de François Étienne en 1765, celle qui montre Marie-Thérèse en pendant de son fils, alors empereur et corégent.

327 Barta, Familienporträts, pp. 33-51. Voir chapitre VI.

328 Voir notamment les chapitres III, IV et V où ce portrait est mentionné. Barta, Familienporträts, pp. 158-160; Schmuttermeier, "Franz Anton Palko », pp. 2-6; Slavíček, " Franz Anton Palko nebo Anton Glunck? ».

329 Baum, «Die Wahl des Erzherzogs Maximilian Franz».

330 Slavíček, « Franz Anton Palko nebo Anton Glunck? », p. 33. 


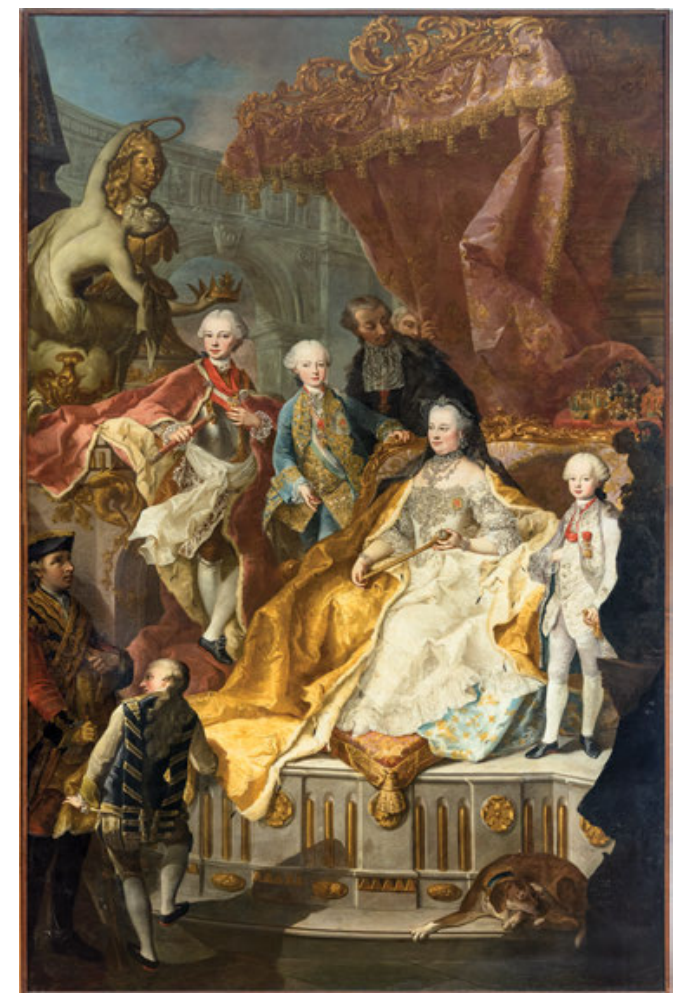

Figure 34: Franz Anton Palko, Marie-Thérèse avec ses plus jeunes fils Léopold, Ferdinand et Maximilien, 1766-1768, 445 × $263 \mathrm{~cm}$, autrefois au couvent prémontré de Louka de Znojmo, aujourd'hui au château de Valtice, République tchèque, VA 360, National Heritage Institute Regional Historic sites management in Kroměřǐž, State château, Valtice.

C’est ainsi que dans ce portrait, exposé au château de Kežmarok (P 165, Figure 35), réalisé par un artiste inconnu, probablement secondaire, en raison de la qualité de l'exécution, Marie-Thérèse veuve est représentée face à son fils Joseph, succédant à la représentation de la souveraine et de son époux. 


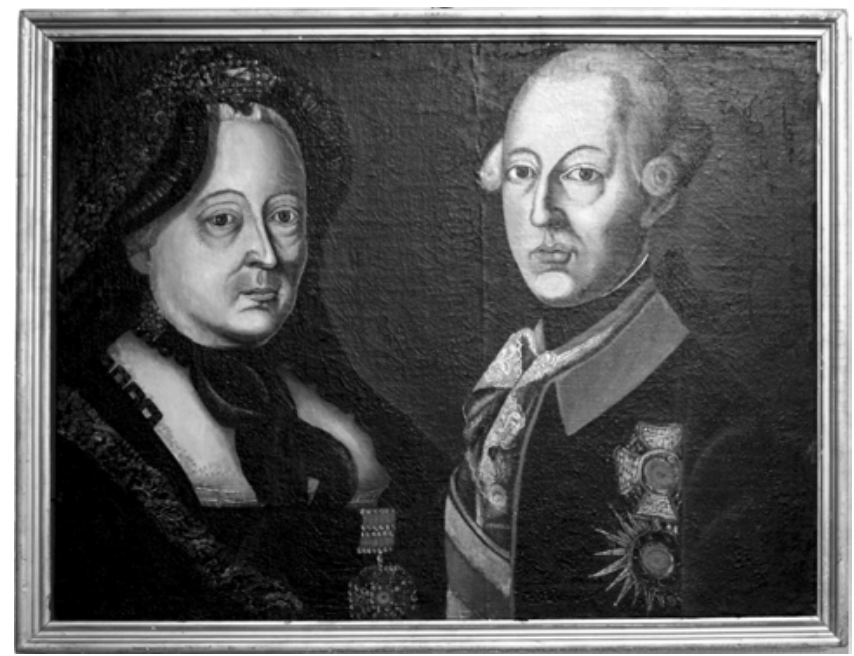

Figure 35: Artiste inconnu, Marie-Thérèse veuve et Joseph II, 50, 6 x 68 cm, château de Kežmarok, Inv. Nr. 10 248, Foto Muzeum v Kežmarku, Slovaquie.

\section{Les Habsbourg-Lorraine : première famille de l'aristocratie}

Tout le complexe iconographique des portraits de famille s'insère dans les traditions de représentation dynastique des Habsbourg, mais il peut également être comparé avec la représentation familiale de la haute noblesse de la Monarchie. Martin van Meytens, avec son atelier, réalise des portraits de famille pour d'autres grands de la Monarchie tels les Schwarzenberg ${ }^{331}$, les Harrach ou les Pálffy. Les nobles commandent auprès des artistes princiers de cour et d'académie des images d'eux-mêmes et de leur descendance ainsi que de leur suzerain et de sa famille, première des familles du royaume. Cette mise en scène familiale est très populaire et répandue à cette époque car les nobles imitent le modèle royal. La famille est en effet un élément central de représentation comme de pouvoir, symbole de continuité. C'est un élément dynastique et nobiliaire par excellence, qui renvoie au besoin des souverains comme des nobles de se créer une lignée. Mais on y trouve aussi des tendances nouvelles, bourgeoises et intimistes à la fois, centrées autour de la famille dite « nucléaire » de nos jours.

Meytens réalise des portraits pour la maison princière des Schwarzenberg ${ }^{332}$. Cette dernière se laisse représenter de la même manière que la famille impériale.

331 Nous renvoyons aussi au chapitre VI où il est déjà question de ces collections aristocratiques. 332 Mörath, «Martin von Meytens ». 
Avant même les grandes commandes impériales des années 1750, Martin van Meytens a déjà pour mission de peindre les autres grandes familles de la Monarchie, dès les années 1740, marquées pour la famille impériale par les portraits de Marie-Thérèse à côté de l'archiduc Joseph. Force est de constater une certaine réappropriation, diffusion et assimilation d'un même modèle de représentation, qui n'est pas spécifique à la dynastie des Habsbourg-Lorraine. Les portraits de famille réalisés par Meytens pour de grandes familles nobles, comme les Schwarzenberg ou encore les Pálffy, sont souvent plus majestueux que les portraits de famille commandés par Marie-Thérèse.

Meytens peint ainsi dans les années 1760 un portrait immense de la famille du comte Miklós Pálffy d'Erdőd. Ce portrait se trouvait jadis dans les collections de la famille et a été légué au Musée du Belvédère ${ }^{333}$. Le comte Pálffy y est représenté aux côtés de son épouse et de cinq de leurs enfants dans un tableau majestueux, sans commune mesure, par sa grandeur et sa qualité artistique, avec le portrait de la famille impériale sur la terrasse du château de Schönbrunn réalisé en plusieurs exemplaires au cours des années 1750. La famille Pálffy est peinte dans un décor champêtre. Le fils du comte, en habit de chasse, montre du doigt son père et le désigne ainsi comme chef de la famille. Ce dernier est peint avec le collier de l'Ordre de la Toison d'Or. Vers 1752 ou 1753, Meytens exécute également un immense portrait de famille, de presque cinq mètres et demi de largeur, pour les Liechtenstein. La famille au complet y est représentée, avec titres et honneurs ${ }^{334}$.

En 1744, année où l'hôtel de ville de Vienne commande le portrait de MarieThérèse avec l'archiduc Joseph enfant, le premier portrait familial commandé par les Schwarzenberg est celui de la famille impériale. Sur ce portrait, qui se trouve exposé dans un salon du château de Neuwaldegg, quatre enfants de MarieThérèse sont représentés assis sur un grand lit jaune dans une salle de marbre : l'archiduchesse Marianne, l'archiduc héritier Joseph qui soulève de la main droite le collier de l'Ordre de la Toison d'Or, ainsi que les archiduchesses MarieChristine et Marie-Élisabeth ${ }^{335}$. Cette commande est suivie deux ans plus tard, en 1746, de celle d'un portrait de la famille Schwarzenberg, renforçant l'idée de familles toutes très liées entre elles. Le prince héritier Schwarzenberg, est représenté de la même manière que l'archiduc Joseph, en costume hongrois et avec le collier de l'Ordre de la Toison d'Or. Les images similaires sont comme des por-

333 Johannsen, « Die Familie des Grafen Nikolaus Pálffy »; voir aussi Barta, Familienporträts, pp. 96-97; Lisholm, Martin van Meytens, pp. 64, 66, 109-110.

334 Voir Kalábová, Konečný, Pretium Laborum non vile, p. 54. 335 Voir le chapitre VI. 
traits d'amis qui entretiennent des relations familières et dont les représentations familiales se font écho par la similitude des thèmes.

Chef d'État, Marie-Thérèse, n'en est pas moins mère et chef de famille. Elle n'est pas la seule souveraine à avoir en main les rênes du pouvoir. La comparaison avec d'autres souveraines comme Élisabeth I ${ }^{\text {ère }}$ d'Angleterre, Christine de Suède ou encore, figure contemporaine de Marie-Thérèse, Catherine de Rus$\operatorname{sie}^{336}$, semble au première abord particulièrement intéressante et riche en matière d'interprétation. Ainsi Catherine de Russie apparaît-elle aussi comme une souveraine à la fois conquérante et éclairée ${ }^{337}$, même si nous verrons dans le chapitre suivant ce qu'il faut entendre par « éclairé » dans le cas de MarieThérèse. Bien qu'elles aient été confrontées aux mêmes enjeux que MarieThérèse (se faire accepter en tant que femmes et chefs politiques à la fois), ces souveraines n'ont jamais été mères de famille comme Marie-Thérèse, à l'exception de Catherine de Russie qui aura un fils, Paul, qui sera tsar après elle. Comme nous l'avons montré, ce rôle de mère de famille est un aspect essentiel de la représentation, et plus particulièrement des portraits de Marie-Thérèse. Cette iconographie est déjà largement développée au Moyen Âge et souligne la maternité de la reine. Être une mère et une vertueuse épouse, irréprochable, est un comportement qui s'appuie sur celui de la Vierge Marie ${ }^{338}$. Cela fait partie d'un type commun et répandu de représentation familiale, privilégié par les peintres. C'est aussi un motif de référence littéraire, largement accepté et diffusé. Épouse et mère, la reine est celle qui garantit et assure la continuité dynastique. Les rôles traditionnels de protection, de justice mais aussi de bonté et de pardon sont répartis entre les deux figures royales ${ }^{339}$. Le roi est représenté comme souverain guerrier et justicier tandis que la reine se doit d'être un symbole de paix, maternel, protecteur et nourricier. De fait, Marie-Thérèse assume

336 Comme le souligne Christina Strunck, les portraits de Marie-Thérèse se distinguent de ceux de Catherine de Russie, en particulier durant le moment de veuvage. En certaines occasions, Catherine imite le geste militaire de Pierre le Grand pour sa propre représentation ; voir Strunck, « The 'Two Bodies' of the Female Sovereign », pp. 72-76.

337 La souveraine russe attire ainsi à sa cour les meilleurs artistes et militaires d'Europe.

338 Cosandey, La reine de France, pp. 282-283 : "Mère et épouse irréprochable : ce code de conduite calqué sur la vie de la Vierge devient un idéal à atteindre. [. . .] », p. 282 ; « Accorder à la reine la figure de Marie revient ainsi à magnifier celle qui, par sa participation dynastique, doit être sans tache et signifier à tous qu'elle a atteint l'idéal de perfection attendue d'une mère de roi », p. 283.

339 Cosandey, « La Blancheur de nos lys », p. 393 : « Au roi de guerre et de victoire fait pendant une reine de paix et de maternité ». 
et est amenée à assumer les deux rôles, guerrière et pacificatrice à la fois ${ }^{340}$. Dans ce contexte, la souveraine apparaît certes comme une mère mais aussi, en quelques cas, comme une guerrière. Le rôle féminin de la souveraine dans ses portraits semble en effet très contrasté.

\section{Une souveraine mère mais aussi guerrière}

L'image d'une reine au combat, victorieuse, met en scène une monarchie et une souveraine triomphante. Par rapport à l'ensemble du corpus, cette pose est exceptionnelle. Il s’agit d'une représentation rare et géographiquement localisée, située dans des provinces de la Monarchie particulièrement menacées durant les guerres de la première moitié du règne. Même si elle ne prend pas personnellement part au combat, Marie-Thérèse n'en est pas moins à sa manière un chef de guerre. Son époux, François Étienne, le fut effectivement quelque temps, de manière toutefois malheureuse. Dans ces quelques portraits équestres, Marie-Thérèse semble s'emparer de cette fonction militaire, l'une des principales missions du souverain mâle, mais d'une manière toute féminine.

La représentation d'une reine victorieuse et celle de l'empereur en chef de guerre sont autant d'arguments pour asseoir la nouvelle dynastie. Qu'importe que de nombreuses batailles ne soient pas gagnées et que la Silésie reste définitivement perdue. Deux tableaux de Marie-Thérèse guerrière et victorieuse se trouvent dans les Pays-Bas autrichiens où c'est un type de portrait assez habituel, une manière traditionnelle de représenter les ducs de Brabant dans la région. Cette représentation équestre est minoritaire au sein du corpus de portraits et la commande ne provient pas de la cour viennoise mais des autorités citadines bruxelloises, comme pour le portrait de Jean-Baptiste Millé (P 84, Figure 36). En matière de représentation équestre, la cour viennoise privilégie les portraits de Marie-Thérèse liés au couronnement hongrois, mettant ainsi en valeur le rituel traditionnel du couronnement hongrois sur la colline de Presbourg, comme nous l'avons vu au cours de cet ouvrage.

340 Fanny Cosandey, pour son objet d'étude, parle de reine « souveraine et maternelle »; Cosandey, « La Blancheur de nos lys », p. 393 ; Fanny Cosandey montre comment l'image diffusée de la reine régente s'appuie sur l'association étroite entre souveraineté et maternité. L'iconographie de Marie de Médicis met en avant la position de la régente. Celle-ci dirige le royaume tout en formant son fils, alors tout jeune roi. C'est bien l'image de la mère et de son fils qui est alors soulignée; voir Cosandey, «La Blancheur de nos lys », p. 394 ; Cosandey, La reine de France, pp. 326-332 ; Germann, Picturing Marie Leszczinska, pp. 23-28. Sur les portraits d'une autre régente, voir Llorente, « Mariana of Austria's Portraits ». 
Les portraits qui se trouvent au sein de l'hôtel de ville de Bruxelles (P 84, Figure 36) et au Musée lorrain de Nancy (P 85, Figure 37) représentent Marie-Thérèse à cheval ${ }^{341}$. Selon nous, ces tableaux, réalisés par des artistes régionaux aux alentours de 1750, au moment de la reconquête des Habsbourg, tant le portrait équestre de l'hôtel de ville de Bruxelles que celui du Musée lorrain, peuvent aussi nous rappeler les compétences guerrières, même limitées, du beau-frère de MarieThérèse, le propre frère de François Étienne de Lorraine, Charles de Lorraine (gouverneur général à Bruxelles pendant trente-six ans, et dès le début très populaire) comme celles, très limitées elles aussi, de l'époux de Marie-Thérèse.

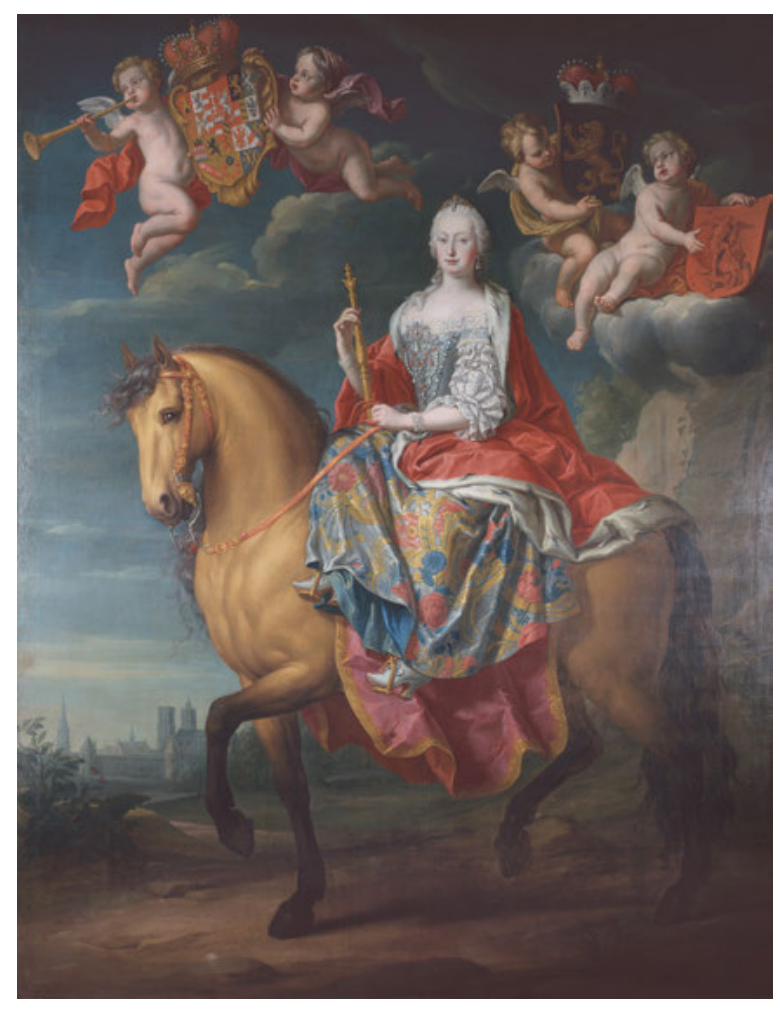

Figure 36: Jean-Baptiste Millé, Marie-Thérèse à cheval, 1750 ou années 1750, 315 x $247 \mathrm{~cm}$, Inv. Nr. K 1750/1, Hôtel de Ville de Bruxelles, Musée de la Maison du Roi, Collection des Musées de la Ville de Bruxelles, Belgique.

341 Nous mentionnons aussi ces portraits dans les chapitres III et V. 
Pour l'aristocratie, le cheval est une représentation symbolique de la noblesse des familles ${ }^{342}$. Le portrait équestre du monarque, motif répandu depuis l'Antiquité, le représente comme un souverain victorieux et conquérant. À l'époque moderne, des peintres comme Titien, avec la représentation de Charles Quint lors de la bataille de Mühlberg ${ }^{343}$, ou encore Pierre Paul Rubens et Anton van Dick, réalisent ce type de portraits pour les souverains et les grands de leur temps. Mentionnons aussi les doubles portraits bien connus de Philippe III d'Espagne et de sa femme Marguerite d'Autriche ainsi que ceux de Philippe IV et de son épouse, tous réalisés par Diego Velázquez pour le palais Buon Retiro à Madrid. Les Bourbons récemment installés sur le trône d'Espagne s'inscrivent dans la lignée et dans la continuité espagnole des Habsbourg. Philippe V se fait ainsi à son tour représenter à cheval par Louis Michel van Loo $^{344}$. Durant le XVIII ${ }^{\mathrm{e}}$ siècle, les hommes d'un rang moins élevé ainsi que les femmes sont de plus en plus souvent peints dans cette posture, comme l'attestent, par exemple, le portrait équestre de Wilhelmine d'Orange, réalisé par Tethart Philipp Christian Haag, ou celui de Marie-Thérèse de Vallabriga, peint par Francisco de Goya, et aujourd'hui conservé aux Offices de Florence ${ }^{345}$.

L'historien Luc Duerloo insiste, quant à lui, sur l'attention accordée aux victoires militaires de l'empereur Charles VI dans le programme iconographique mis en place lors de l'investiture bruxelloise de $1717^{346}$. Bien qu'il doive très vite gouverner en formant un partenariat, selon la métaphore de Duerloo, avec les différents états provinciaux des Pays-Bas autrichiens, l'empereur Charles VI se présente et est représenté comme un homme victorieux et confiant, et qui ne semble confronté à aucune opposition ${ }^{347}$. Quelques décennies plus tard, dans les années 1750, après la reconquête autrichienne sur les troupes françaises, l'hôtel de ville de Bruxelles fait de nouveau réaliser un portrait équestre du monarque victorieux, cette fois Marie-Thérèse.

Dans le portrait équestre de Marie-Thérèse conservé à l'hôtel de ville de Bruxelles (P 84, Figure 36), la souveraine arrive face au spectateur, triomphante, même si

342 Concernant l'importance matérielle et symbolique du cheval, voir notamment l'ouvrage de Roche, La culture équestre.

343 Checa Cremades, Carlos V., p. 271.

344 C'est une huile sur toile de 345 x 264 cm, conservée au Palais de la Granja de San Ildefonso à Ségovie. Voir entre autres l'ouvrage de Soler del Campo, The art of power : Royal Armor and Portraits from Imperial Spain, pp. 162-163, pp. 170-171, pp. 190-191, pp. 202-232, pp. 202-203 et pp. 208-210. Sur la carrière de Van Loo, voir Rolland, « Louis Michel Van Loo » et « Louis Michel Van Loo et les académies au XVIII ${ }^{\mathrm{e}}$ siècle ».

345 Pons, « Gemälde von Gedanken leer », pp. 236-239.

346 Duerloo, « Discourse of Conquest », pp. 474-475.

347 Duerloo, « Discourse of Conquest », pp. 475-476; Van Gelder, « The Investiture of Emperor Charles VI », p. 444. 
son cheval est peint de profil, à l'image d'une souveraine victorieuse, comme semblent l'annoncer les petits amours ou putti, dont l'un joue de la trompette, proclamant la renommée de la souveraine. Ces derniers portent des couronnes, dont le chapeau archiducal, ainsi que les écus aux armes du Brabant, des Flandres et des armoiries de Bruxelles. Ce tableau de grandes dimensions inscrit Marie-Thérèse dans une représentation royale d'inspiration flamande, avec en arrière-plan une vue de la ville de Bruxelles, notamment l'église Sainte-Gudule et l'hôtel de ville. Dans ce portrait, Marie-Thérèse est représentée en amazone, de façon féminine et gracieuse. Le cheval peut ici faire figure de trône et c'est bien un sceptre que MarieThérèse tient dans ses mains de manière presque délicate, comme si elle touchait de sa main un éventail. Au Musée lorrain de la ville de Nancy, un portrait très similaire au portrait bruxellois, représente en effet Marie-Thérèse à cheval (P 85,

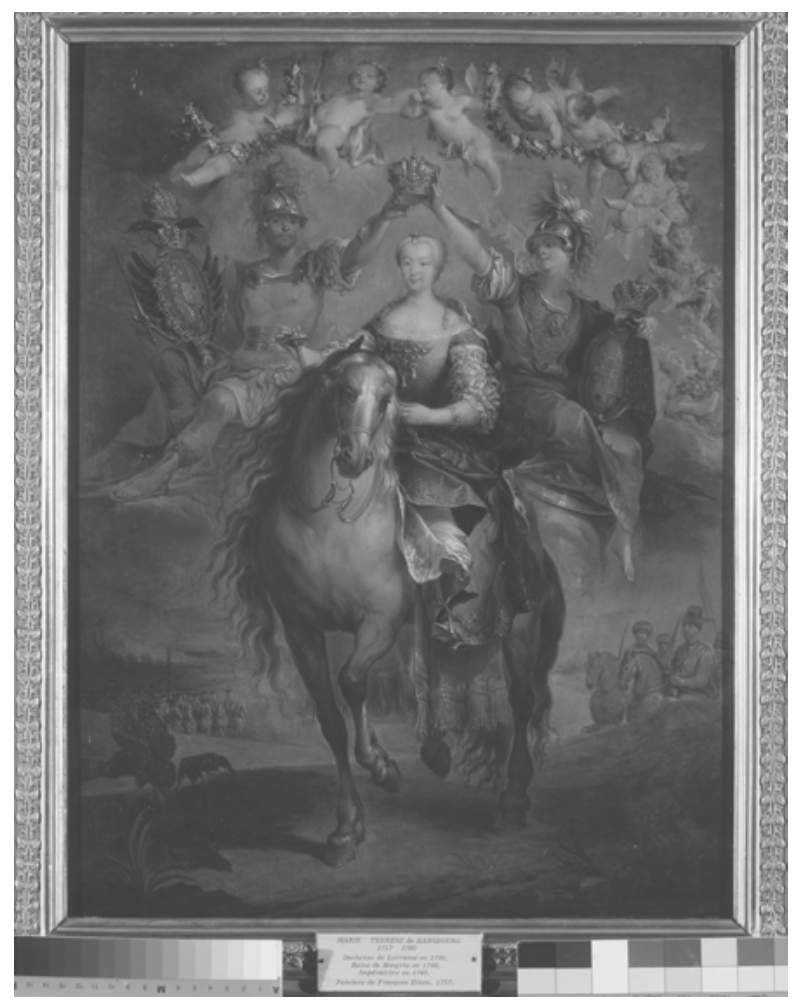

Figure 37: François Eisen, portrait équestre de l'impératrice Marie-Thérèse de Habsbourg, épouse de François Étienne de Lorraine, ancien duc de Lorraine, Huile sur bois, 1757, 57,7 x 43,2 cm, Inv. 72.2.4. ( ) Palais des ducs de Lorraine-Musée Lorrain, Nancy /Photo. G. Mangin, France. 
Figure 37$)^{348}$. Ce portrait est daté de 1757, il est également réalisé par un artiste originaire des Pays-Bas autrichiens, François Eisen. La différence majeure est la taille, le portrait lorrain, une huile sur bois, est de dimensions beaucoup plus réduites que le portrait bruxellois.

Dans le portrait de François Eisen, les soldats qui ressemblent à des magnats hongrois sont peints en arrière-plan, rappelant ainsi les activités guerrières de la Monarchie et l'aide apportée par les Hongrois à la souveraine en début du règne. Ici, Marie-Thérèse est toujours représentée en amazone, elle apparaît cependant à la façon d'un chef de guerre, mêlant habilement, comme dans le portrait de Bruxelles, victoire et féminité. L'allure du cheval, qui arrive de face, est victorieuse. La souveraine se dirige frontalement, triomphante, par rapport au spectateur. Des personnages, en armure, lui font une haie d'honneur et tiennent ce qui semble être une couronne impériale familiale au-dessus de la tête de la souveraine, les armoiries des Flandres sont représentées de chaque côtée ${ }^{349}$. Des troupes apparaissent au fond du tableau et font certainement allusion à l'invasion des troupes françaises au sein des Pays-Bas autrichiens. Les lauriers et les arcs de triomphes, présents dans les deux portraits du couple impérial, prennent toute leur signification; la figure de Minerve penchée sur eux ${ }^{350}$.

Achetés le 9 mars 1972 au Palais Galliera, les tableaux de Marie-Thérèse et de François Étienne de Lorraine sont signés et datés en bas à droite Fois (François) Eisen 1757 le père et sont préemptés pour le compte du Musée lorrain, ce qui explique leur inscription dans les registres du Louvre ${ }^{351}$. Il existe une grande similitude de présentation entre ce portrait équestre de Marie-Thérèse au Musée lorrain et la Prise de Juliers (Louvre) de Pierre Paul Rubens ${ }^{352}$, l'allure du cheval notamment est très similaire dans ces deux tableaux. Cela peut être lu comme un témoignage de l'influence de la galerie Marie de Médicis de Paris. Le recueil gravé de la Galerie du Luxembourg à Paris a certainement servi d'intermédiaire ; des puttis ou petits anges sont repris de l'Heureux Gouvernement de Rubens $^{353}$. La proximité entre Paris et Bruxelles peut expliquer que des peintres

348 Vilain, «Peintures de l'école française », pp. 352-353.

349 Voir aussi l'autre portrait de François Eisen, Portrait équestre de François III en empereur, Huile sur bois, 1757, Inv. 72.2.3. Musée Lorrain, Nancy/photo.G. Mangin.

350 Le portrait de François Étienne de Lorraine fait pendant au portrait de son épouse. Badach, Stanislas, un roi de Pologne en Lorraine, p. 113; Franz, Laumon, Martin, François de Lorraine, pp. 20-21.

351 Vilain, « Peintures de l'école française », pp. 352-353, p. 352.

352 Voir notamment le portrait de Pierre Paul Rubens sur Le triomphe de Juliers, 394 x 295 cm, Huile sur toile, 1622-1625, Paris, Musée du Louvre.

353 Voir Vilain, « Peintures de l'école française », pp. 352-353, 352, et comme il nous a été aimablement indiqué par le Musée lorrain de Nancy qui s'appuyait sur la Revue du Louvre et 
d'influence flamande, plutôt que des artistes autrichiens ou hongrois, aient privilégié ce type de portraits équestres, différents de la représentation traditionnelle du couronnement hongrois. Remarquons que la représentation de Marie-Thérèse au sein des Pays-Bas autrichiens (P 84, Figure 36) n'est pas, elle non plus, sans rappeler « l'heureux gouvernement », la fresque du flamand Rubens réalisée en l'honneur de Marie de Médicis. Le portrait équestre de Marie-Thérèse, commandé par les autorités de la ville de Bruxelles, offre une représentation plus originale que la plupart des autres portraits de la région et des autres territoires héréditaires.

Bruxelles notamment témoigne ainsi de son rattachement et de son attachement à Marie-Thérèse, représentée à cheval, selon un motif traditionnel du souverain (mais aussi de la souveraine) en chef de guerre. Ce motif iconographique peut aussi être lu comme une marque de loyauté vis-à-vis de la Monarchie, alors si bien représentée par Charles de Lorraine à Bruxelles et dans ces provinces périphériques de la Monarchie.

\section{Conclusion}

L'image du souverain sort renforcée de la confrontation avec la figure féminine dont la maternité est le principal attribut. Les quinze dernières années du règne offrent cependant une autre facette de cette féminité en représentant la souveraine veuve et femme avant tout. Marie-Thérèse est le chef d'une famille nombreuse mais aussi un chef politique sorti finalement victorieux après de nombreuses années de guerre.

Les véritables critères qui permettent de répondre à la question de la féminité présumée de Marie-Thérèse tiennent à l'analyse du corps naturel de MarieThérèse, et surtout de sa place en tant qu'épouse mais aussi comme mère. La féminité, comprise principalement sous les termes de fertilité et de maternité, n'est plus un obstacle et peut être utilisée à des fins politiques ${ }^{354}$.

Si la Monarchie est ainsi légitimée grâce aux couronnements réussis de Marie-Thérèse et de son époux ainsi que par leur nombreuse descendance, la mort inattendue de François Étienne en 1765 bouleverse cependant considérablement Marie-Thérèse et modifie sa représentation. Son apparence change. De nouveaux insignes du pouvoir sont peints. La souveraine veuve s’appuie désor-

des Musées de France précédemment citée. Nous remercions Claire Tiné et Bénédicte Pasques du Service de documentation du Musée lorrain de Nancy.

354 Strunck, "The 'Two Bodies' of the Female Sovereign », p. 76: « Maria Theresia's body natural became her body politic as she turned her motherhood into a political argument in support of her claim to the preservation and protection of her dynasty ». 
mais davantage sur l'exemple féminin de sa mère et de sa grand-mère. Cette image de veuve, d'impératrice veuve, donne à son portrait une force nouvelle.

$\mathrm{Au}$ cours de ce chapitre, nous avons tenté de montrer les divers aspects de la question du genre dans les portraits de Marie-Thérèse. Il est évident que l'aspect le plus essentiel de cette question du genre est la maternité. C'est un véritable argument politique dans ce contexte d'extinction de la lignée masculine des Habsbourg en 1740. Les enfants du couple impérial sont sans cesse mis en avant $^{355}$, notamment dans les tableaux. Le rôle spécifiquement féminin de la maternité n'empêche pas Marie-Thérèse d'être et de rester le souverain. À cette question du genre, rattachons la représentation de la souveraine à cheval. Il s'agit d'une image assez rare au sein de notre corpus de tableaux. Ce type de portraits se rattache toutefois à un genre déjà proposé pour des femmes, comme l'attestent les fresques réalisées en l'honneur de Marie de Médicis par Rubens.

Ces deux faces de la représentation - mère et en apparence guerrière - offrent une image spécifique, bien que contrastée, de Marie-Thérèse : celle de la mère chef d’État.

355 La «prospérité » des Habsbourg-Lorraine est sans cesse présentée en public ; voir Braun, Eine Kaiserin und zwei Kaiser, p. 159. 